\title{
Plant Pattern Analysis To Increase Agricultural Harvest Production In Taebenu District, West Timor, Indonesia
}

\author{
Sutirto $^{1}$, Yunus Fallo ${ }^{1}$, Arnoldus Nama ${ }^{1}$ \\ \{sutirtojatayu@gmail.com,yunus_fallo@yahoo.co.id,n.arnold.Id@gmail.com\} \\ State Polytechnic of Kupang, Indonesia
}

\begin{abstract}
The Tulun Irrigation Network is an area of 200 hectares of irrigated agricultural area, the source of water in the Tulun irrigation network comes from the Fefanaek Dam. The Tulun irrigation channel consists of primary and secondary canals with a total length of $1430.47 \mathrm{~m}$. In this study, an analysis of irrigation water needs was analyzed, analysis of irrigation water availability, analysis of water balance, and optimization of the Tulun Irrigation Area. The results of the water balance indicate that the availability of water is not sufficient to meet water needs based on existing cropping patterns. Results of analysis Establishment of cropping patterns according to water requirements to irrigate rice fields in order to increase agricultural output in the Tulun irrigation area of Taebenu District is rice - palawija (second crops in dry season) - palawija which started from the preparation of land in November 1 starting in January 2 .
\end{abstract}

Keywords: planting pattern, production, harvest, agriculture, irrigation, optimization

\section{Introduction}

Increasing the water demand in the context of intensification and expansion of rice fields, as well as a limited water supply for irrigation and other needs, especially in the dry season, the distribution and use of irrigation must be carried out more efficiently and effectively [1].

The government's goal to achieve a just, prosperous and prosperous Indonesian society is the noble ideals of the Indonesian Nation as enshrined in the Preamble of the 1945 Constitution [2]. This needs to be supported by several factors, including natural resources and human resources. One of the current government targets is to make Indonesia a food selfsufficiency, country so that various government programs are launched to achieve this goal, even remote and disadvantaged areas are striving to develop such as the Province of East Nusa Tenggara (NTT).

President Joko Widodo said the problem of East Nusa Tenggara (NTT) was only water. According to the President, the development of NTT depends on the availability of water. If there is water, the community can use it to develop the agricultural potential such as corn. Communities in the East Nusa Tenggara (NTT) region have a variety of livelihoods, one of which is farming. Most farmers use irrigation water to meet water needs in rice fields, dry field farming, animal husbandry and fisheries. Generally, water is obtained from irrigation facilities and infrastructure built by the government or the farmers themselves. The NTT region is one area that is always experiencing drought and lack of water. To answer this, water management is very necessary in order to meet the needs of the community. The government 
is trying to develop irrigation areas of dryland or wetland that has the potential to be developed into agricultural land that can meet the needs of the community (Tempo, 2016)

One of the districts in NTT that often experiences drought problems is Kupang Regency. In supporting water needs in the agricultural sector with an irrigation system, there will indeed be several problems that arise. The availability of irrigation water in the dry season still lacks water and vice versa during the rainy season abundant water that is not used optimally.

In Taebenu Subdistrict, Kupang Regency, there are rice fields, the majority of the people are farmers, each year the agricultural output is decreasing every year because it always constrains water shortages. The area of agriculture is $200 \mathrm{Ha}$ with a length of $1,430.7 \mathrm{~m}$ of primary and secondary channel infrastructure.

The existence of the above problems requires a study of cropping pattern analysis to improve agricultural output:

1. How is the application of plant pattern according to the water requirements to irrigate the rice fields to increase agricultural output in the Tulun irrigation area of Taebenu District.

2. What is the required debit in the intake channel according to the results of the analysis of the plant pattern schedule in order to meet the debit needed in the Tulun Irrigation Network to irrigate 200 hectares of rice fields.

\section{Research Methods}

\subsection{Research Location}

The research location was the Tulun irrigation network in Taebenu District, West Timor, Indonesia. Geographically, the location of the study is located at coordinates $10^{\circ} 10^{\prime} 56.92^{\prime \prime}$ latitude and 123 ${ }^{\circ} 0^{\prime} 56.60^{\prime \prime}$ longitude. With territorial boundaries i.e. Northern with Central Kupang District, Eastern with East Baumata Village, Western with West Baumata Village, and Southern with Central Baumata Village.

The surface condition of the land in the village of North Baumata includes flat and low surface types. The climate in this region is a semi-arid climate with a dry season longer than the rainy season. The dry season is from April to October, while the rainy season is from November to March.

Increasing the water demand in the context of intensification and expansion of rice fields, as well as a limited water supply for irrigation and other needs, especially in the dry season, the distribution and use of irrigation must be carried out more efficiently and effectively.

The government's goal to achieve a just, prosperous and prosperous Indonesian society is the noble ideals of the Indonesian Nation as enshrined in the Preamble of the 1945 Constitution. This needs to be supported by several factors, including natural resources and human resources. One of the current government targets is to make Indonesia a food selfsufficiency, country so that various government programs are launched to achieve this goal, even remote and disadvantaged areas are striving to develop such as the Province of East Nusa Tenggara (NTT).

President of Indonesia said the problem of East Nusa Tenggara (NTT) was only water. According to the President, the development of NTT depends on the availability of water. If there is water, the community can use it to develop the agricultural potential such as corn. Communities in the East Nusa Tenggara (NTT) region have a variety of livelihoods, one of which is farming. Most farmers use irrigation water to meet water needs in rice fields, dry 
field farming, animal husbandry and fisheries. Generally, water is obtained from irrigation facilities and infrastructure built by the government or the farmers themselves. The NTT region is one area that is always experiencing drought and lack of water. To answer this, water management is very necessary in order to meet the needs of the community. The government is trying to develop irrigation areas of dryland or wetland that has the potential to be developed into agricultural land that can meet the needs of the community (Tempo, 2016)

One of the districts in NTT that often experiences drought problems is Kupang Regency. In supporting water needs in the agricultural sector with an irrigation system, there will indeed be several problems that arise. The availability of irrigation water in the dry season still lacks water and vice versa during the rainy season abundant water that is not used optimally.

In Taebenu Subdistrict, Kupang Regency, there are rice fields, the majority of the people are farmers, each year the agricultural output is decreasing every year because it always constrains water shortages. The area of agriculture is $200 \mathrm{Ha}$ with a length of $1,430.7 \mathrm{~m}$ of primary and secondary channel infrastructure.

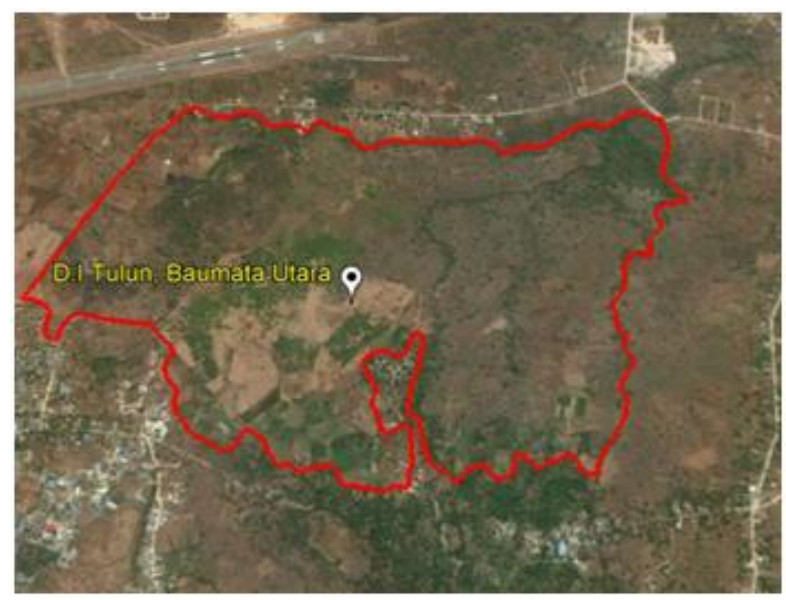

Fig. 1. Research Location

\subsection{Procedure of Research}

The initial step in this research is collecting references used as a basis for research related to water resources, then hydrological analysis is carried out [3]. One of the important hydrological parameters in a work related to water resources is to calculate available water discharges, calculate the water requirements needed in a rice field by means of alternative cropping patterns, with the following steps:

1. 10-year Rainfall Data from (BMKG Lasiana Kupang Climatology Station) Station Climatology station adjacent to the Research Area;

2. Climatology Data of the last 10 years consisting of temperature, solar radiation, humidity, wind speed, area of irrigated rice fields, water availability analysis;

3. Calculate the average amount of $1 / 2$ monthly rainfall;

4. Calculating potential evapotranspiration;

5. Calculate percolation and infiltration;

6. Analysis of the schedule calculation by means of shifting crops, according to the availability of the land and determining the types of plants to be planted; 
7. Determine the flow of water at the intake (intake) according to the calculation results of shifting cropping patterns.

\section{Results And Discussion}

\subsection{Irrigation Water Needs}

\section{Water Needs in Rice Fields}

Calculation of Water Needs for Land Preparation (S). Calculation of water requirements for land preparation in November:

Evapotranspiration $(\mathrm{ETo})=4,104 \mathrm{~mm} /$ day

Percolation $(\mathrm{P})=2.00 \mathrm{~mm} /$ day

Number of Exponents $(\mathrm{e})=2.7182$

Land Preparation Period $(\mathrm{T})=45$ days

Water requirements for fulfillment $(\mathrm{S})=300 \mathrm{~mm}$

Water requirements to replace water losses due to evaporation and percolation in rice fields:

$\mathrm{M}=1.1 \times \mathrm{ETo}+\mathrm{P}=1.1 \times 4.104+2.00$

$=6,514 \mathrm{~mm} / \mathrm{day}$

$\mathrm{k}=\mathrm{Mx}=6,514 \mathrm{x}$

$=0.977$

$\mathrm{ek} \quad=(2.7182) 0.977=2.657$

$\mathrm{LP}=(\mathrm{M} \mathrm{x} \mathrm{ek}) /(\mathrm{ek}-1)=(6,514 \times 2,657) /(2,657-1)$

$=10.446 \mathrm{~mm} /$ day.

\subsection{Mainstay Rainfall (R80)}

To calculate the water needs in a rice field, the daily rainfall data are calculated into semi-monthly data. Data was obtained from several rainfall stations around the Tulun Irrigation Area research site. The rainfall station is included in the Baumata Watershed (DAS) catchment area. The rainfall stations in the Baumata River Basin are Lasiana Climatology Station and Eltari Meteorological Station. Daily rainfall data have been calculated into semimonthly rainfall data for the two rainfall stations above.

\subsection{Calculation of Effective Rainfall (Re)}

Effective rainfall for rice plants is calculated based on $70 \%$ of the mainstay rainfall value of $80 \%$ [4], while for effective rainfall for crops and sugarcane is calculated based on the value of the mainstay rainfall of 50\%. This calculation was taken in November as an example calculation. The results of the calculation of Effective Rainfall (Re) are presented in Table 1. 
Table 1. Calculation of Effective Rainfall (Re)

\begin{tabular}{|c|c|c|c|c|c|}
\hline \multirow{2}{*}{ Bulan } & \multirow{2}{*}{ R80 } & $\mathrm{Re}=0.7 * \mathrm{R} 80$ & Re Padi & $\mathrm{Re}=0.5 * \mathrm{R} 80$ & Re Palawija \\
\hline & & $(\mathrm{mm})$ & $(\mathrm{mm} / \mathrm{hr})$ & $(\mathrm{mm})$ & $(\mathrm{mm} / \mathrm{hr})$ \\
\hline \multirow{2}{*}{ Jan } & 51.000 & 35.700 & 2.380 & 25.500 & 1.700 \\
\hline & 178.940 & 125.258 & 8.351 & 89.470 & 5.965 \\
\hline \multirow{2}{*}{ Feb } & 54.220 & 37.954 & 2.530 & 27.110 & 1.807 \\
\hline & 51.740 & 36.218 & 2.415 & 25.870 & 1.725 \\
\hline \multirow{2}{*}{ Mar } & 60.040 & 42.028 & 2.802 & 30.020 & 2.001 \\
\hline & 47.240 & 33.068 & 2.205 & 23.620 & 1.575 \\
\hline \multirow{2}{*}{ Apr } & 0.720 & 0.504 & 0.034 & 0.360 & 0.024 \\
\hline & 0.200 & 0.140 & 0.009 & 0.100 & 0.007 \\
\hline \multirow{2}{*}{ Mei } & 0.060 & 0.042 & 0.003 & 0.030 & 0.002 \\
\hline & 0.000 & 0.000 & 0.000 & 0.000 & 0.000 \\
\hline \multirow{2}{*}{ Jun } & 0.000 & 0.000 & 0.000 & 0.000 & 0.000 \\
\hline & 0.000 & 0.000 & 0.000 & 0.000 & 0.000 \\
\hline \multirow{2}{*}{ Jul } & 0.000 & 0.000 & 0.000 & 0.000 & 0.000 \\
\hline & 0.000 & 0.000 & 0.000 & 0.000 & 0.000 \\
\hline \multirow{2}{*}{ Ags } & 0.000 & 0.000 & 0.000 & 0.000 & 0.000 \\
\hline & 0.000 & 0.000 & 0.000 & 0.000 & 0.000 \\
\hline \multirow{2}{*}{ Sep } & 0.000 & 0.000 & 0.000 & 0.000 & 0.000 \\
\hline & 0.000 & 0.000 & 0.000 & 0.000 & 0.000 \\
\hline \multirow{2}{*}{ Okt } & 0.000 & 0.000 & 0.000 & 0.000 & 0.000 \\
\hline & 0.000 & 0.000 & 0.000 & 0.000 & 0.000 \\
\hline \multirow{2}{*}{ Nov } & 1.200 & 0.840 & 0.056 & 0.600 & 0.040 \\
\hline & 17.890 & 12.523 & 0.835 & 8.945 & 0.596 \\
\hline \multirow{2}{*}{ Des } & 51.920 & 36.344 & 2.423 & 25.960 & 1.731 \\
\hline & 121.250 & 84.875 & 5.658 & 60.625 & 4.042 \\
\hline
\end{tabular}

Source: Calculation Results of This Research

\subsection{Irrigation Water Availability}

Calculation of availability of irrigation water using the F. J. Mock method with the required data as follows:

1) Semi-monthly rainfall (mm)

2) Semi-monthly rainy days

3) Potential Evapotranspiration Value (ETo)

4) River Basin Area (DAS)

For example the calculation of Mainstay Debit by the F. J. Mock method for January 12009 is as follows:

Watershed Area $=7,414 \mathrm{~km} 2$

1. Rain Data

- Semi-monthly rainfall

- P $=78 \mathrm{~mm} / 15 \mathrm{hr}$

- Half-monthly rainy days $h=9$ days

2. Limited Evapotranspiration (Et)

3. Semi-monthly potential evapotranspiration

ETo $=$ ETo $\times 15$ days, $=3,076 \times 15$ days $=46,134 \mathrm{~mm} / 15$ days

Open land surface $(\mathrm{m})=40 \%$ (intended for cultivated agricultural land assuming $\mathrm{m}=30 \%$ $50 \%) .(\mathrm{m} / 20) \times(18-\mathrm{h})=(40 \% / 20) \times(18-9)=0.180 . \mathrm{E}=($ ETo $) \times(\mathrm{m} / 20) \times(18-\mathrm{h})=$ $46,134 \times 0,180=8,304 \mathrm{~mm} / 15 \mathrm{hr}$

Difference between Potential Evapotranspiration (ETo) and Limited Evapotranspiration (Et) $\mathrm{Et}=\mathrm{ETo}-\mathrm{E}=46,134-8,304=37,830 \mathrm{~mm} / 15 \mathrm{hr}$

\subsection{Water Balance}

1) Rainwater that reaches the ground surface $\Delta \mathrm{s}=\mathrm{P}-\mathrm{Et}$ 
$=78-37,830$

$=40,170 \mathrm{~mm} / 15 \mathrm{hr}$.

2) Groundwater content (Is)

If the value of $\Delta s>0$, the moisture content of water in the soil is 0 . Conversely, if $\Delta s<0$, the amount of moisture content of water in the soil is the value of $\Delta \mathrm{s}$ itself. This means that if the price positifs is positive $(\mathrm{P}>\mathrm{Et})$ then water will enter the soil if the soil's moisture capacity has not been met, and vice versa will run out if the soil is saturated. If the price negatifs is negative $(\mathrm{P}<\mathrm{Et})$ then some ground water will come out and there will be a deficit. In January $12008, \mathrm{P}>\mathrm{Et}$ so $\Delta \mathrm{s}>0$. Therefore the amount of soil moisture content in January 12008 was $0 \mathrm{~mm} / 15 \mathrm{hr}$.

3) Soil moisture capacity (SMC)

Determine soil moisture capacity (SMC) parameters [5]. The initial SMC value in January of the first period was estimated at $250 \mathrm{~mm}$. For the next month / period depends on the value of the moisture content of the water in the soil. If the value is negative, the amount of SMC in the next month / period is the difference from the previous month / period SMC value and the value of padas in the following month. SMC values are usually taken from 50 to $250 \mathrm{~mm}$.

4) Excess water (WS)

$\mathrm{WS}=\Delta \mathrm{s}-\mathrm{Is}=40,170-0,000=40,170 \mathrm{~mm} / 15 \mathrm{hr}$.

5) Groundwater Flow and Storage

The infiltration coefficient (i) is estimated based on soil porosity conditions and the slope of the drainage area [6]. Porous land such as fine sand has a higher infiltration than heavy clay soils. Steep land where water does not have time to infiltrate into the soil then the coefficient of infiltration will be small. The infiltration coefficient limit is $0-1.0$. The infiltration coefficient $(\mathrm{i})$ is taken $=0.8$. Recession factor groundwater flow $(\mathrm{k})=0.9$.

6). Infiltration (I)

$\mathrm{I}=\mathrm{WS} \times \mathrm{I}=40.170 \times 0.8=32.136 \mathrm{~mm} / 15 \mathrm{hr}$

$0.5 \times(1+\mathrm{k}) \times \mathrm{I}=0.5 \times(1+0.9) \times 32,136=30,529$

7) $\mathrm{kx} \mathrm{V}(\mathrm{n}-1)=0.9 \times 415,686=374,117$

7) Storage volume $(\mathrm{Vn})$

$\mathrm{Vn}=0.5(1+\mathrm{k}) \mathrm{I}+\mathrm{k}(\mathrm{V}(\mathrm{n}-1))$

$=30,529+374,117$

$=404,647 \mathrm{~mm} / 15 \mathrm{hr}$

8) Changes in water volume $(\Delta \mathrm{Vn})$

$\Delta \mathrm{Vn}=\mathrm{Vn}-\mathrm{V}(\mathrm{n}-1)=404,647-415,686$

$=-11,039 \mathrm{~mm} / 15 \mathrm{hr}$

9) Basic flow (BF)

$\mathrm{BF}=\mathrm{I}-\Delta \mathrm{Vn}=32,136-(-11,039)=43,175 \mathrm{~mm} / 15 \mathrm{hr}$

10) Direct flow (DR)

$\mathrm{DR}=\mathrm{WS}-\mathrm{I}=40,170-32,136=8,034 \mathrm{~mm} / 15 \mathrm{hr}$

11) Flow (R)

$\mathrm{R}=\mathrm{BF}+\mathrm{DR}=43,175+8,034=51,209 \mathrm{~mm} / 15 \mathrm{hr}$

12) River Flow Discharge

River flow discharge $=($ AxRx1000 $) /(86400 \times$ Number of days $)=(7,414 \times 51,209 \times$ $1000) /(86400 \times 15)=0.293 \mathrm{~m} 3 / \mathrm{sec}=292,952 \mathrm{lt} / \mathrm{sec}$

After knowing the amount of irrigation water needs that have been calculated based on 6 alternatives and the amount of water available that irrigates the Tulun Irrigation Area, it can be 
seen how the potential of the water meets the irrigation water needs in the Tulun Irrigation Area by calculating planting intensity as follows:

Taken an example of calculation January 1 alternative 1

Where: NFR $=0.463 \mathrm{lt} / \mathrm{sec} / \mathrm{ha}, \mathrm{Q}$ Mainstay $=0.150 \mathrm{~m} 3 / \mathrm{sec}$, Irrigation Area $=200 \mathrm{ha}$

$\mathrm{Q}$ Need $=$ NFR $\mathrm{x}$ Irrigation Area

$$
=0.463 \times 200=92,575 \mathrm{lt} / \mathrm{sec}
$$

$\mathrm{Q}$ Mainstay $=0.150 \times 1000=149,800 \mathrm{lt} / \mathrm{sec}$

Irrigation Area $=(\mathrm{Q}$ Mainstay/ $\mathrm{Q}$ Need $) \mathrm{x}$ Irrigation Area

$$
=(92,575 / 149,800) \times 200=323,628 \text { ha }
$$

The results of the calculation of cropping intensity can be seen in Tables 6 and 7 below. Furthermore, from the calculation of cropping intensity it can be seen the largest cropping intensity of the six alternatives available. The highest planting intensity is the optimal planting start time.

Table 2. Calculation of Monthly Mainstay Discharge from 2009 - 2018 using the F. J. Mock

\begin{tabular}{|c|c|c|c|c|c|c|c|c|c|c|c|c|c|c|c|c|c|c|c|c|c|c|c|c|c|}
\hline \multirow[b]{2}{*}{ Tahn } & & & \multicolumn{2}{|c|}{$\frac{V x}{x}$} & \multicolumn{2}{|c|}{4} & \multicolumn{2}{|c|}{ Wha } & \multicolumn{2}{|c|}{ Jin } & & \multicolumn{2}{|c|}{ 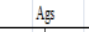 } & \multicolumn{2}{|c|}{ Sep } & \multicolumn{2}{|c|}{ Oit } & \multicolumn{2}{|c|}{ Nor } & \multicolumn{2}{|c|}{ Des } & \multirow{2}{*}{$\begin{array}{l}\text { Total } \\
\text { ahnnan }\end{array}$} \\
\hline & 1 & & 1 & 2 & 1 & 2 & 4 & & & 2 & 1 & & & & 1 & , & 1 & & 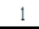 & 2 & 1 & & 1 & 2 & \\
\hline & $\mathrm{m}^{3}$ tati & the & & $\mathbb{m}^{3}$ & titi & $\mathrm{m}^{3} / 4$ & the & $\left.m^{3}\right)^{3}$ the & dit. & $\mathrm{m}^{3} \mathrm{~d}_{\mathrm{a}}$ & 3 tith & $\mathrm{m}^{3} / \mathrm{d} t \mathrm{ti}$ & this & dat & $\mathrm{m}^{3} / \mathrm{dth}$ & $\mathrm{m}^{3} \mathbf{H}^{3}$ & dit. & titis & id & mithe & $\mathrm{m}^{3} / \mathrm{dt}$ & tet & titi & tots & $\mathrm{n}^{3}$ dat \\
\hline 2000 & 0293 & & & 1.048 & & 0.552 & & 48 & 003 & 0.34 & 316 & 0.299 & 64 & 223 & 214 & & 173 & 6 & 140 & 0.119 & 14 & 153 & 392 & 300 & 6.650 \\
\hline 2010 & & & & & & & & & & & & & & & & & & & & & & & 328 & & \\
\hline 2011 & 249 & & & & & & & & & & & & & & & & & & & & & 100 & 181 & & 1.9 \\
\hline 2012 & 6.1 & & & & & & & & & & & & & & & & & & & & & & 220 & 225 & 1506 \\
\hline 2013 & & & & & & r & & & & & & & & 0.160 & & & 0.122 & 12. & 101 & & 082 & 128 & 144 & 0.277 & 6.67? \\
\hline 2014 & & & & & & 0 & & & & & & 0.7 & & 0.216 & & & 0.168 & & & & & & 2066 & 0.247 & 3255 \\
\hline 2013 & & & & & & & & & & & & 0. & & & & & & & & & & & & & \\
\hline 2016 & 0.569 & & & $(x+2)$ & 0.605 & & & & & & & 0. & & 0.159 & & & & & 等 & & & & 19 & 20, & \\
\hline 2017 & 0.10 & 0.287 & 0.228 & 0.192 & 0294 & 0.18 & 0.167 & 0.150 & 15 & 0.135 & 0.129 & 0.1 & 15 & 0.0 & 180 & 0.0 & 0.069 & 62 & 56 & 0.047 & 131 & 186 & 179 & 0.206 & \\
\hline 2018 & 019 & 0.62 & 0.638 & 0.470 & 0.413 & 0.327 & & & & & 0208 & 0.187 & & 0142 & 0.136 & 0.115 & 111 & 999 & 090 & 0.132 & 0.092 & 0.175 & 153 & 0.202 & \\
\hline
\end{tabular}
Method

Source: Calculation Results of This Research

Table 3. Resume of Water Needs with Alternative Planting Patterns Planting Pattern: Rice Palawija - Palawija

Irrigation Area Area: 200

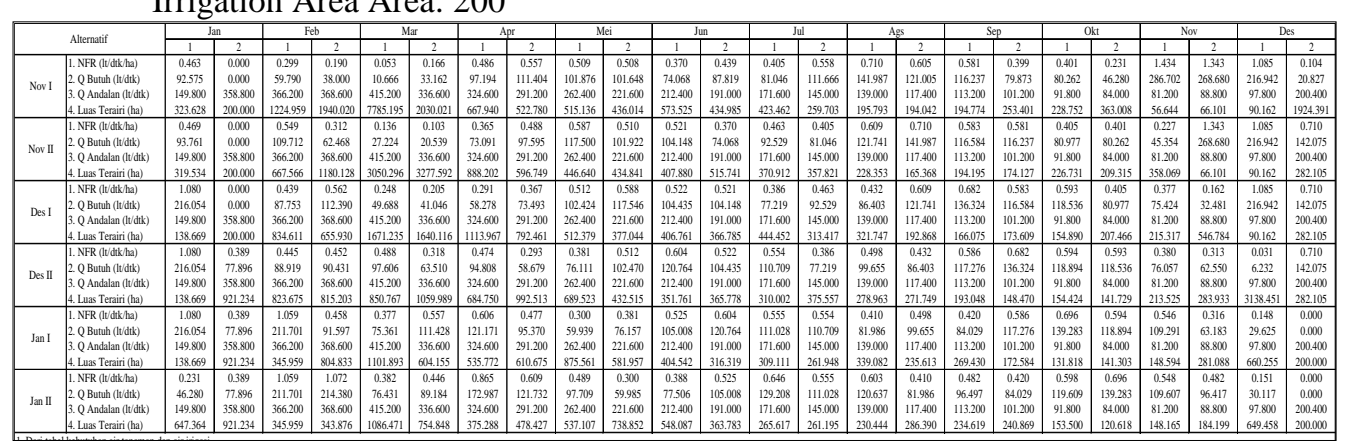

Source: Calculation Results of This Research 
Table 4. Water Needs and Maximum Area Acrossable for Alternatives Nov I - Jan 2 Planting Pattern: Rice - Palawija - Palawija Irrigation Area: 200 ha

\begin{tabular}{|c|c|c|c|c|c|c|c|c|c|c|c|c|c|c|c|c|c|c|c|c|c|c|c|c|c|}
\hline \multirow{3}{*}{ Alt } & \multirow{2}{*}{\begin{tabular}{|l|l|l|} 
Bulan \\
Periodele
\end{tabular}} & \multicolumn{2}{|c|}{ 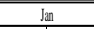 } & \multicolumn{2}{|c|}{ Feb } & \multicolumn{2}{|c|}{ Mar } & \multicolumn{2}{|c|}{ Apr } & \multicolumn{2}{|c|}{ Mei } & \multicolumn{2}{|c|}{ Jun } & \multicolumn{2}{|c|}{$\begin{array}{lll}\text { Jul } \\
\text {. }\end{array}$} & \multicolumn{2}{|c|}{ Aggs } & \multicolumn{2}{|c|}{ Sep } & \multicolumn{2}{|c|}{ Okt } & \multicolumn{2}{|c|}{ Nor } & \multicolumn{2}{|c|}{ Des } \\
\hline & & 1 & 2 & 1 & 2 & 1 & 2 & 1 & 2 & 1 & 2 & 1 & 2 & 1 & 2 & 1 & 2 & 1 & 2 & 1 & 2 & 1 & 2 & 1 & \\
\hline & 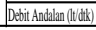 & 149.900 & 358.800 & 3662200 & 368.6000 & 415.200 & 336.6000 & 324.600 & 29.1200 & 262.400 & 221.600 & 2124.400 & 199.1000 & 177.600 & 145.000 & 139,000 & 117.40 & 1132200 & 10.2000 & 99.800 & 84,000 & 81.200 & 88.800 & 97.800 & 200.400 \\
\hline & & & & & & & & & & & & & & & & & & & & & & & & & \\
\hline & QButuh (Itralik) & 22575 & 0.000 & \begin{tabular}{|l|l}
59.900 \\
\end{tabular} & 38.000 & 10.666 & 33.162 & \begin{tabular}{|l|}
97.194 \\
\end{tabular} & 111.404 & \begin{tabular}{|l|l|}
10.8976 \\
\end{tabular} & 101.648 & 74.668 & 87.819 & 81.046 & 111.666 & 141.987 & 121.005 & \begin{tabular}{|l|l|}
116.237 \\
\end{tabular} & 79.873 & 80.162 & 6.280 & 6.702 & 68.680 & 16.942 & 20.827 \\
\hline $\mathrm{N}_{0}$ & Luas Terari (ha) & 200.000 & 200.000 & 200.000 & 200.000 & .000 & 200.000 & 200.000 & 200.000 & 200.000 & 200.000 & 200.000 & 200.000 & 200.000 & 200.000 & 195.793 & 194.022 & 194.774 & 200.000 & 200.000 & 200.000 & 56.644 & 66.101 & 90.162 & 200.000 \\
\hline & Q Butuh (Itudilk) & 93.761 & 0.000 & 109.712 & 62468 & 27224 & 20.539 & 73.091 & 97595 & 1175000 & 101922 & 104.148 & 74.688 & 92525 & 81.046 & 121.741 & 141.987 & 116.584 & 116.237 & 80.977 & 80.262 & 45.354 & 266.680 & 266942 & 142.2075 \\
\hline Nov II & Luas Terari (tha) & 200.000 & 200.000 & 200.000 & 200.000 & 200.000 & 20.000 & 200.000 & 200.000 & 200.000 & 200.000 & 200.000 & 200.000 & 200.000 & 200.000 & 200.000 & 165.68 & 194.195 & 174.127 & 200.000 & 200.000 & 200.000 & 66.101 & 90.162 & 200.000 \\
\hline & Q Butuh (It/didk) & 216.654 & 0.000 & 87753 & 1123,30 & 49.688 & 41.066 & 58.278 & 73.493 & 102424 & 117.546 & 104435 & 100.148 & 77.219 & 92529 & 86.413 & |21.7.74 & 136.24 & 116.584 & \begin{tabular}{|l|l|}
118.536 \\
\end{tabular} & 80977 & 75.24 & 32481 & 216942 & 1422075 \\
\hline Des & Luas Terairi (ha) & 138.699 & 200.000 & 200.000 & 200.000 & 200.000 & 200.000 & 200.000 & 200.000 & 200.000 & 200.000 & 200.000 & 20.000 & 200.000 & 200.000 & 200.000 & 128688 & 166.075 & 173.609 & 155.800 & 200.000 & 200.000 & 200.000 & 90.162 & 200.000 \\
\hline & QBunh (Ifrdilk) & 216.654 & 778.896 & \begin{tabular}{|l|l|l|l|}
88919 \\
\end{tabular} & 99.431 & \begin{tabular}{|l|}
97.606 \\
\end{tabular} & 63.510 & \begin{tabular}{|l|l|}
94,808 \\
\end{tabular} & 58.679 & \begin{tabular}{|l|l|}
76.111 \\
\end{tabular} & 102470 & 120.764 & 104.435 & 110.709 & 77.219 & 99.655 & 86.403 & \begin{tabular}{|l|l|}
117.276 \\
\end{tabular} & 136.324 & \begin{tabular}{|l|l|}
118.894 \\
\end{tabular} & 118.536 & 76.057 & 62550 & 6.322 & 1422.075 \\
\hline & Luas Terairit (tha) & 138.699 & 200.000 & 200.000 & 200.000 & 200.000 & 20.000 & 200.000 & 200.000 & 200.000 & 200.000 & 200.000 & 200.000 & 200.000 & 200.000 & 200.000 & 200.000 & 193.48 & 148.470 & 154.424 & $\mid 141.729$ & 200.000 & 200.000 & 200.000 & 200.000 \\
\hline & Q Bunuh (Itith & 216.654 & 778986 & \begin{tabular}{|l|l|}
21.701 \\
\end{tabular} & 91:597 & \begin{tabular}{|l|l|}
75.361 \\
\end{tabular} & 111.428 & \begin{tabular}{|l|l|}
121.171 \\
\end{tabular} & \begin{tabular}{|l|l|}
95370 \\
\end{tabular} & \begin{tabular}{|l|l|}
59.939 \\
\end{tabular} & \begin{tabular}{|l|}
76.157 \\
\end{tabular} & \begin{tabular}{|l|l|}
105.008 \\
\end{tabular} & 1200.764 & 111.028 & 110.709 & 81986 & 99.655 & \begin{tabular}{|l|}
84,029 \\
\end{tabular} & 117.276 & \begin{tabular}{|l|l|}
139.283 \\
\end{tabular} & \begin{tabular}{|l|l|}
118.894 \\
\end{tabular} & 109.291 & 63.183 & 29.625 & 0.000 \\
\hline Jat & Luas Terariri (tha) & 138.699 & 200.000 & 200.000 & 200.000 & 200.000 & 200.000 & 200.000 & 200.000 & 200.000 & 200.000 & 200.000 & 20.000 & 200.000 & 200.000 & 200.000 & 200.000 & 200.000 & 172.584 & \begin{tabular}{|l|l|}
131.818 \\
\end{tabular} & $\mid$\begin{tabular}{|l}
14.303 \\
\end{tabular} & 148.54 & 200.000 & 200.000 & 200.000 \\
\hline & Q Bundh (Itidili & 46.280 & 77.896 & 211.701 & 214,380 & 76.431 & 89.184 & 172987 & 122.732 & \begin{tabular}{|l|}
97.709 \\
\end{tabular} & 59.985 & 775.56 & 105.008 & 1292200 & 111.028 & 120.637 & 88.996 & \begin{tabular}{|l|}
96.497 \\
\end{tabular} & 84.029 & 199.609 & 139.283 & 109.607 & 96.417 & 30.177 & 0.000 \\
\hline & Lus Terairif (tha) & 200.0000 & 200.000 & 200.000 & 200.000 & 200.000 & 200.000 & 200.000 & 200.000 & 200.000 & 200.000 & 200.0000 & 20.000 & 200.000 & 200.000 & 200.000 & 200.000 & 200.000 & 200.000 & 53.500 & 120.618 & 148.165 & 184.199 & 200.000 & 200.000 \\
\hline
\end{tabular}

Source: Calculation Results of This

Table 5. Planting intensity in Tulun Irrigation Area

\begin{tabular}{|c|c|c|c|c|c|c|c|c|c|c|}
\hline \multirow{2}{*}{ ALT } & \multirow{2}{*}{ Bulan } & \multicolumn{2}{|c|}{ Musim Tanam I } & \multicolumn{2}{|c|}{ Musim Tanam II } & \multicolumn{2}{|c|}{ Musim Tanam III } & \multirow{2}{*}{$\begin{array}{c}\text { Total } \\
\text { Luas (Ha) }\end{array}$} & \multirow{2}{*}{ Rencana } & \multirow{2}{*}{ Prosentase $(\%)$} \\
\hline & & NFR Max & $\mathrm{A}(\mathrm{Ha})$ & NFR Max & $\mathrm{A}(\mathrm{Ha})$ & NFR Max & $\mathrm{A}(\mathrm{Ha})$ & & & \\
\hline ALT 1 & Nov I & 1.434 & 56.644 & 0.557 & 200.000 & 0.710 & 194.042 & 450.686 & 200 & 225 \\
\hline ALT 2 & Nov II & 1.343 & 66.101 & 0.587 & 200.000 & 0.710 & 165.368 & 431.469 & 200 & 216 \\
\hline ALT 3 & Des I & 1.085 & 90.162 & 0.588 & 200.000 & 0.682 & 154.890 & 445.052 & 200 & 223 \\
\hline ALT 4 & Des II & 1.080 & 138.669 & 0.604 & 200.000 & 0.682 & 141.729 & 480.398 & 200 & 240 \\
\hline ALT 5 & Jan I & 1.080 & 138.669 & 0.604 & 200.000 & 0.696 & 131.818 & 470.488 & 200 & 235 \\
\hline ALT 6 & Jan II & 0.696 & 120.618 & 1.072 & 200.000 & 0.646 & 200.000 & 520.618 & 200 & 260 \\
\hline \multirow{2}{*}{$\begin{array}{l}\text { Mulai } \\
\text { Tanam }\end{array}$} & Pola Tata & \multicolumn{2}{|c|}{ Tanam I } & \multicolumn{2}{|c|}{ Tanam II } & \multicolumn{2}{|c|}{ Tanam III } & \multirow[b]{2}{*}{$\begin{array}{c}\text { Total Luas } \\
\text { (Ha) }\end{array}$} & \multirow{2}{*}{$\begin{array}{c}\text { Areal } \\
\text { Potensial } \\
(\mathrm{Ha})\end{array}$} & \multirow{2}{*}{$\begin{array}{c}\text { Intensitas } \\
\text { Tanam } \\
(\%)\end{array}$} \\
\hline & Tanam & $\begin{array}{l}\text { NFR Max } \\
(\mathrm{l} / \mathrm{d} / \mathrm{ha})\end{array}$ & $\begin{array}{l}\text { Luas } \\
(\mathrm{Ha})\end{array}$ & $\begin{array}{c}\text { NFR Max } \\
(\mathrm{lt} / \mathrm{d} / \mathrm{ha})\end{array}$ & $\begin{array}{l}\text { Luas } \\
\text { (Ha) }\end{array}$ & $\begin{array}{l}\text { NFR Max } \\
\text { (It/dt/h) }\end{array}$ & $\begin{array}{l}\text { Luas } \\
\text { (Ha) }\end{array}$ & & & \\
\hline Jan II & Pd-Plw-Plw & 0.696 & 120.618 & 1.072 & 200.000 & 0.646 & 200.000 & 520.618 & 200 & 260 \\
\hline
\end{tabular}

Source: Calculation Results of This Research

Limited amount of water in the dry season can reduce water supply to rice fields [7]. To maximize farm production, it is necessary to increase land productivity and provide regular water supply according to needs and supplies. For this analysis a linear program Quantity Methods for Windows 3 is used with input water requirements for each type of plant and the mainstay volumes as constraints for the operation of the linear program [8]. The output of this program is the maximum area of rice fields for each type of crop, the growing season and the benefits of the farm [9]. From the several alternative plans, cropping patterns obtained that produce the greatest benefits are cropping patterns of sugarcane, rice-crops-sugarcane, sugarcrops at the beginning of November 1 with a profit of $\operatorname{Rp} 281,541,700,000.00$ and cropping intensity 300 (Wahyudi, et al. 2014).

The condition of the water system in the study area is not completely good. The planting pattern in the study area is only once planting season in a year (rice) [10], while the planned planting pattern is three times planting season per year (rice-rice-palawija) and the maximum water demand occurs in October the second period of $261.35 \mathrm{~mm} /$ period or $17.42 \mathrm{~mm} /$ day. Secondary and tertiary sluice operation patterns of lowland rice plants during the rainy season focus on retention, controlled drainage to remove excessive rainwater or during fertilization, flushing and washing of toxic and acidic elements, and tidal irrigation [11]. Drainage is needed if the puddle becomes too deep or if the water quality deteriorates. For secondary crops planted in the dry season after harvesting the second rice crop is finished focusing on drainage. From the results of the analysis of the farming business, it is obtained that the prediction of profit for the current cropping pattern in the study area (Rice) is Rp 1,495,000 /ha/year and for the planned cropping pattern (Rice-Rice-Palawija) is Rp 7,730,750/ha/year [12]. 


\section{Conclusion}

Based on the analysis results above, the following conclusions can be drawn:

1. The application of plant pattern according to water needs to irrigate rice fields to increase agricultural output in the Tulun irrigation area of Taebenu District is rice - Palawija Palawija which starts from November 1 to January 2. In that month there was still a surplus, while in July to December the river water flow deficit.

2. Water intake at the largest intake is needed according to the results of the analysis of the plant pattern schedule to irrigate 200 hectares of rice fields in the Tulun Irrigation Network of 1,434 Lt/Sec/ha. The availability of incoming water discharge at Intake is in accordance with the analysis starting with the planting pattern from November to January, amounting to $2,205 \mathrm{Lt} / \mathrm{Sec}$.

\section{Recomendation}

a) There are 4 parameters that can be changed to optimize the availability of water, namely planting season, class system, tertiary plot rotation coefficient, and planting start time. In this study, optimization is carried out by changing the start time of planting. So that in subsequent studies changes can be made to other parameters.

b) Communities must be more efficient in using and utilizing water.

c) Need for regular channel maintenance by P3A.

d) The community is more concerned with planting patterns that have been determined.

e) It is recommended that before conducting research it is better to prepare all the necessary data so that the preparation does not experience difficulties.

\section{References}

[1] Anonimous, 2006. Peraturan Pemerintah Republik Indonesia No 20 Tahun 2006, Tentang Irigasi, Jakarta

[2] Anonimous, 2010, Direktorat Jenderal Departemen Pekerjaan Umum. Standar Perencanaan Irigasi-Kriteria Perencanaan 01. Badan Penerbit Departemen Pekerjaan Umum. Jakarta

[3] Asdak, 2010. Hidrologi dan Pengelolaan Daerah Aliran Sungai. Gadjah Mada University, Jogyakarta

[4] Linsley, Ray K.JR., Kohler, M.A. and Paulus, J.L.H. 1989. Hidrologi Untuk Insinyur. Terjemahan Hermawan. Penerbit Erlangga Jakarta

[5] Mangostina, C, 2010. Pengertian Curah Hujan, Pernerbit Graha ilmu, Yogjakarta

[6] Mochammad, B. 2013. Irigasi. Graha Ilmu, Yogjakarta

[7] Montarcih, L. (2010). Hidrologi Praktis. Penerbit Lubuk Agung, Bandung

[8] Sasrodarsono, S. 1977. Hidrologi Untuk Pengairan. Pradnya Paramita. Jakarta

[9] Soemarto, CD. 1995,. Hidrologi Teknik. Penerbit Erlangga Jakarta

[10] Wahyudi A., Anwar N. and Ediyatno 2014 Studi Optimasi Pola Tanam pada Daerah Irigasi Warujayeng Kertosono dengan Program Linier. Journal of Teknik Pomits Vol. 3 No. 1: $77-83$ 
[11] Wirosudarmo R. and Apriadi U. 2010. Studi Perencanaan Pola Tanam Dan Pola Operasi Pintu Air Jaringan Irigasi Reklamasi Rawa Pulau Rimau di Kabupaten Musi Banyuasin Sumatra Selatan. Jourrnal of Teknologi Pertanian Vol.3 No.1: 56 - 66. 